\title{
Approach to Diagnosis and Treatment of Allergy to Alternaria alternata in Patients with Chronic Obstructive Pulmonary Disease and Perennial Allergic Rhinitis
}

\author{
Alexander P. Nazarenko*; G.I. Nazarenko; A.G. Kuznetsov \\ The P.L. Shupyk National Medical Academy of Postgraduate Education (NMAPE); \\ Clinic of Immunology and Allergology "Forpost" \\ Kyiv, Ukraine
}

\begin{abstract}
The aim of our study was to determine the degree of sensitization to Alternaria alternata in patients with chronic obstructive pulmonary disease (COPD) and perennial allergic rhinitis (PAR), and evaluate the effectiveness of allergen-specific immunotherapy (SIT) in patients sensitized to Alternaria alternata.

Material and Methods: All patients were divided into two groups. Group 1 included 130 patients (53 women and 77 men) with COPD aged from 24 to 50 years. Group 2 included 162 patients ( 90 women and 72 men) with PAR aged from 15 to 46 years. The allergen-specific IgE to fungi of the genera: Alternaria alternata, Aspergillus fumigatus, and Penicillinum notatum, as well as the allergen-specific serum IgG antibodies to Alt a 1 of Alternaria alternata were determined. To perform subcutaneous SIT, we used purified major Alt a 1 allergen of Alternaria alternate.

Results: Our study showed that specific IgE antibodies to Alt a 1 were found in $38 \%$ and $44.6 \%$ patients with COPD and PAR, respectively. SIT induced the IgG response against Alt a 1. The concentration of specific IgG antibodies to Alt a 1 increased approximately 8 -fold in COPD patients and 15-fold in PAR patients after 8 months of treatment.
\end{abstract}

Keywords: Chronic obstructive pulmonary disease (COPD); perennial allergic rhinitis (PAR); Alternaria alternate; allergenspecific immunotherapy (SIT).

\section{Introduction}

Respiratory allergies are increasing worldwide. People are exposed to aeroallergens in various settings, both at home and at work. Fungi are ubiquitous airborne allergens and are important causes of human diseases, especially in the upper and lower respiratory tracts. It is estimated that approximately $2-6 \%$ of the general population in developed countries is allergic to fungi [1]. Mostly sensitivity is detected to genera of Alternaria alternata, Cladosporium herbarum, Aspergillus fumigatus, Penicillium spp and Fusarium. Alternaria alternata is one of the most important allergenic molds found in Europe [2]. Alternaria alternata is one of the most common outdoor

*Corresponding author: Alexander P. Nazarenko, Director of Clinic of Immunology and Allergology "Forpost"; Kyiv, Ukraine. E-mail:director@forpost.ua molds, but also has been found in the indoor environment. Dampness and mold problems have been reported to occur in $20 \%$ to $50 \%$ of modern homes [3-5].

Alternaria alternata is known to be a problem in allergic disease. Studies have shown that up to $70 \%$ of mold-allergic patients have skin test reactivity to Alternaria alternata. Allergy to fungi often appears as type I immediate, IgEmediated hypersensitivity, which manifests various allergic diseases, such as bronchial asthma, most types of sinusitis, allergic rhinitis, and pollinosis. Many studies showed that increased serum total $\operatorname{IgE}$ is also a sensitive marker for chronic obstructive pulmonary disease (COPD) patients with higher smoking index, longer duration of illness, more severe lung function impairment [6-9]. COPD is characterized by persistent airflow limitation, and is a major cause of morbidity and mortality worldwide. COPD is a heterogeneous disease and can be classified into different "phenotypes" [10]. A recent study of Jamieson et al. [11] showed that there was an 
"allergic phenotype" of COPD, which accounted for $21 \%$ or $30 \%$ by allergy history (doctor-diagnosed hay fever or allergic symptoms) or allergy testing (increased allergen-specific IgE) in two cohorts.

A high level of $\mathrm{IgE}$ antibodies is one of the risk factors for development of COPD according to Standards for the diagnosis and treatment of patients with COPD (Table 1) [12].

Table 1. Risk factors for chronic obstructive pulmonary disease

\begin{tabular}{|c|c|}
\hline Host factors & Exposures \\
\hline $\begin{array}{l}\text { Genetic factors } \\
\text { Sex } \\
\text { Airway hyperreactivity, } \\
\quad \text { IgE and asthma }\end{array}$ & $\begin{array}{l}\text { Smoking } \\
\text { Socio-economic status } \\
\text { Occupation } \\
\text { Environmental pollution } \\
\text { Perinatal events and childhood illness } \\
\text { Recurrent bronchopulmonary infections } \\
\text { Diet }\end{array}$ \\
\hline
\end{tabular}

Study of G. Rohde et al. [13] showed elevated IgE antibodies directed against $S$. aureus enterotoxins (SAE) in the serum of patients with COPD. Production of IgE to SAE is of non-atopic origin, but rather reflects the superantigen activity on B- and T-cells. This data indicate an immunological reaction to superantigens as a possible trigger of chronic inflammation in COPD, which needs further study.

In addition, sensitization to molds is also a reason for development of perennial allergic rhinitis (PAR) $[3,5,14]$. Therefore, the investigation of sensitization to infectious agents including molds is the actual problem in the study of the pathogenesis of COPD and PAR.

The use of allergen-specific immunotherapy (SIT) to treat the mold sensitized patients one of the most debated aspects. The use of purified protein allergens takes a particular place, which implies determination of their components and evaluation immunotherapy by determining the specific antibodies to the purified allergens.

In this regard, the aim of our study was to determine the degree of sensitization to Alternaria alternata in patients with COPD and PAR, and evaluate the effectiveness of SIT in patients sensitized to Alternaria alternata.

\section{Material and Methods}

All patients were divided into two groups. Group 1 included 130 patients (53 women and 77 men) with COPD aged from 24 to 50 years. The differential diagnosis of COPD was performed according to standard criteria [14]. Group 2 included 162 patients (90 women and 72 men) with PAR aged from 15 to 46 years. PAR diagnosis was verified according to standards of diagnosis based on the clinical manifestation of the disease, allergic anamnesis, and data of the quantitative level of allergen-specific IgE. The control group constituted 20 healthy, age-matched, randomly selected persons.

The study samples were the blood serum of patients. The allergen-specific IgE to fungi of the genera: Alternaria alternata, Aspergillus fumigatus, and Penicillinum notatum, as well as the allergen-specific serum IgG antibodies to the protein Alt a 1 of Alternaria alternata were determined.
The investigation of allergen-specific serum $\operatorname{IgE}$ and $\operatorname{IgG}$ antibodies was performed by immunofluorescence method using ImmunoCAP system («Phadia AB», Sweden).

To perform subcutaneous SIT, we used purified major Alt a 1 allergen of Alternaria alternata of «Diater» (Spain) production. The injections were performed in the presence a physician and in clinic that was equipped to manage possible life-threatening reactions. An assessment of the patient's current health status was made before the administration of immunotherapy injections to determine whether there have been any recent changes in the patient's health that may require modifying or withholding treatment (e.g., exacerbation of allergy symptoms). The subcutaneous injections of an allergen-containing extract were carried out according to the scheme.

During the build-up phase, the injections were performed weekly during the month:

Concentration \#1: $0.1 \mathrm{ml}$, then $0.2 \mathrm{ml}$ after 30 minutes; Concentration \#2: $0.4 \mathrm{ml}$, then $0.4 \mathrm{ml}$ after 30 minutes;

Concentration \#3: $0.1 \mathrm{ml}$, then $0.2 \mathrm{ml}$ after 30 minutes;

Concentration \#4: $0.4 \mathrm{ml}$ and $0.4 \mathrm{ml}$ after 30 minutes or $0.8 \mathrm{ml}$ in one stage.

During the maintenance phase, the injection of $0.8 \mathrm{ml}$ of allergen-containing extract was performed monthly during 7 months; the total number of injections was 8 . The treatment continued for a period of 8 months.

The study was conducted in accordance with ethical principles of the Declaration of Helsinki. It was approved by the local Ethics Committees. Written informed consent was obtained from all participants.

Results were statistically processed using the software package Statistica 6.1 for Windows. The mean (M) and standard error of the mean (SEM) were deduced. Student's unpaired and paired t-tests were used to compare two groups for data with normal distribution. $P$ values of $<0.05$ were considered statistically significant.

\section{Results and Discussion}

We found the increased serum total $\operatorname{IgE}$ in COPD/ PAR patients: $68.9 \pm 11.4 / 185.4 \pm 28.3 \mathrm{kU} / 1$ vs. $16.4 \pm 5.7 \mathrm{kU} / 1$ in the control group $(\mathrm{P}<0.01)$. The presence of specific IgE antibodies to fungal allergens was detected in $42.2 \%$ of COPD patients. The sensitization to Alternaria alternata and Aspergillus fumigatus was detected most frequently (Fig.1).

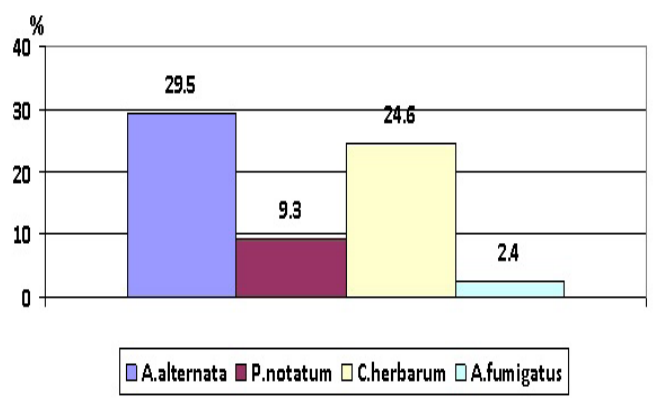

Fig.1. The frequency of allergen specific $\operatorname{IgE}$ antibodies to molds in COPD patients 
The next step in our study was to determine the serum level of allergen-specific $\mathrm{IgE}$ antibodies in PAR patients. The analysis of the spectrum of sensitization in PAR patients showed that the sensitization to the indoor allergens, especially to dust mites and epidermal allergens of pets was identified most frequently. In particular, sensitization to the indoor allergens was detected in $75.6 \%$ of patients, among which the dust mites were dominated; sensitization to fungal allergens was detected in $24.4 \%$ of patients. Mostly sensitivity was detected to genera of Alternaria alternate (60\%), Penicillium notatum (8\%), Cladosporium herbarum (13\%), and Aspergillus fumigates (19\%), Fig.2.

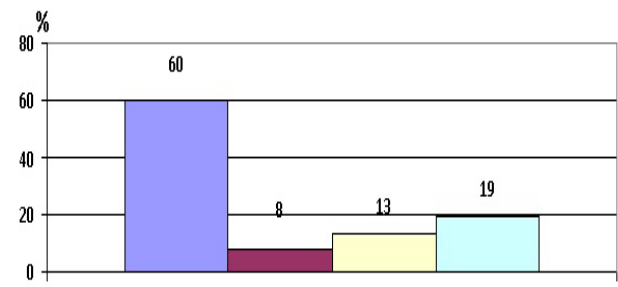

$\square$ A.alternata $\square$ P.notatum $\square$ C.herbarum $\square$ A.fumigatus

Fig. 2. The frequency of allergen specific $\operatorname{IgE}$ antibodies to molds in PAR patients

Because of a high percentage of sensitization to molds, SIT was conducted in COPD/PAR patients sensitized to Alternaria alternata. To determine the genuine sensitization to Alternaria alternata, we identified specific IgE antibodies to Alt a 1, the major Alternaria allergen. Currently, total of 11 allergenic extracts of Alternaria alternata are identified, although only one of them is the most common. Alt a 1 causes the production of specific IgE antibodies in more than $90 \%$ of Alternaria alternata - sensitized patients $[2,4,15]$. Our study showed that specific IgE antibodies to Alt a 1 were found in $38 \%$ of patients with COPD; the genuine sensitization to Alternaria alternata was not confirmed in $4.2 \%$ of cases, and levels of specific IgE antibodies to Alt a 1 were within normal limits.

Studies suggest that before starting SIT need to identify a causative protein that causes allergic reactions and, accordingly, determine the presence of sensitization to the major allergens and exclude the minor components which cause cross-reactivity.

All patients sensitized to Alt a 1 underwent SIT with using a highly purified Alt a 1 produced by «Diater» (Spain). The treatment continued for a period of 8 months. Currently in Europe, the quality of commercial fungal extracts is unstable. In this regard, the purified mold allergens have a great interest, since the purified allergens can be produced in the appropriate conditions of purity and stability for each batch and, therefore, can be a fully standardized diagnostic material. Control and effectiveness of the treatment was determined by assessing the patient's condition. The presence of chronic cough and dyspnea on exertion in COPD patients, as well as nasal congestion and rhinorrhea in PAR patients were registered. In addition, the serum levels of specific anti- Alt a $1 \mathrm{IgE}$ and $\mathrm{IgG}$ antibodies were monitored. As is known, the specific IgG antibodies block the development of allergic reactions, and, according to recent studies, the blocking antibodies appear in form of IgG4 and IgG1-antibodies [15].

Figure 3 shows the results obtained in patients of Group 1. At the end of the 1 st month of treatment, we observed a slight improvement in clinical status of patients (Fig.3a). Chronic cough decreased only in $1 \%$ of cases, and dyspnea on exertion in $3 \%$ of cases. However, at the end of the 4 th month of SIT, chronic cough already mentioned only in $62 \%$ of patients, which was $25 \%$ less than its initial value. Dyspnea on exertion was observed in $36 \%$ of patients, which was $17 \%$ less than its initial value. At the end of therapy, morning cough persisted in only $17 \%$ of patients, dyspnea on exertion in $12 \%$ of patients.

The serum level of specific IgE antibodies continued to increase by $6 \%$ (on average) per month until the end of five months of treatment (Fig.3b). Thus, before SIT, the serum level of specific IgE antibodies to Alt a 1 was $85.4 \pm 4.9 \mathrm{kU} / 1$ in COPD patients, and at the end of 5 months of treatment it increased up to $110 \pm 13.2 \mathrm{kU} / 1(P<0.01)$. Before SIT, the serum level of specific IgG antibodies to Alt al was $2.1 \pm 0.2 \mathrm{kU} / 1$, and at the end of 5 months of treatment it increased by almost 3 times and reached $5.6 \pm 1.2 \mathrm{kU} / 1$. At the end of 6 months of treatment, we observed a decrease in the level of specific IgE antibodies, and his level was $26.7 \pm 8.2 \mathrm{kU} / 1(P<0.01)$ to SIT completion, while the level of specific IgG antibodies continued to rise, and at the end of treatment it was $15.9 \pm 4.3 \mathrm{kU} / 1(P<0.01)$, which was about 8 times higher as compared with baseline values (Fig.3c).
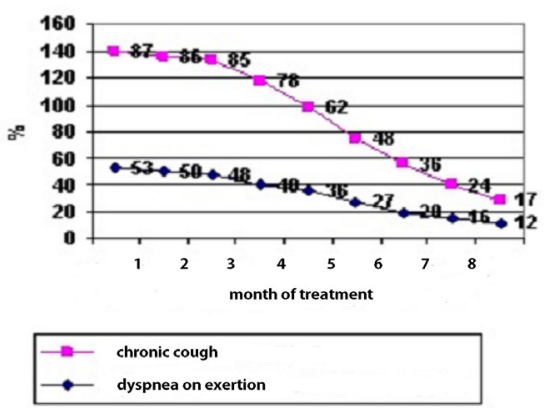

(a)

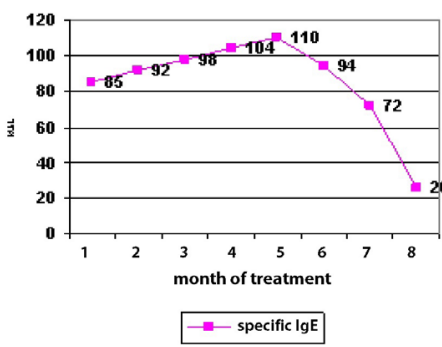

(b)

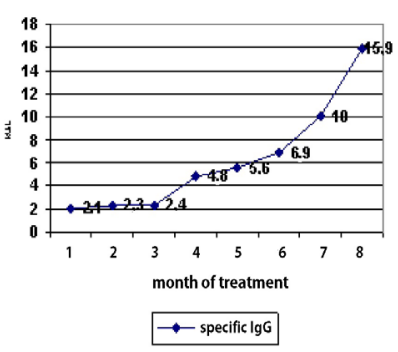

(c)
Fig.3. Dynamics of clinical manifestations (a), the level of specific $\lg E(b)$ and $\operatorname{Ig} G(c)$ antibodies to Alt a 1 during SIT in COPD patients

Specific IgE antibodies to Alt a 1 were found in $44.6 \%$ of Group 2 patients; the genuine sensitization to Alternaria 
alternata was not confirmed in $5.4 \%$ of cases. Control and effectiveness of the treatment was determined by assessing the clinical manifestation and the serum levels of specific anti-Alt a1 $\mathrm{IgE}$ and $\operatorname{IgG}$ antibodies were also monitored. Figure 4 shows the results obtained in patients of Group 2. At the end of the 3rd month of SIT, nasal congestion remained in $52 \%$ of PAR patients, which was $32 \%$ less than its initial value; rhinorrhea decreased to $25 \%$, which was $30 \%$ less than its initial value (Fig.4a). During the next three months of treatment, we have defined a significant reduction in complaints. After 6 months of treatment, nasal congestion and rhinorrhea persisted only in $20 \%$ and $10 \%$ of PAR patients, respectively. At the end of SIT, number of patients with signs of clinical improvement continued to increase. At the end of the 8 th month of treatment, nasal congestion persisted only in $15 \%$ of patients and was less pronounced; the less pronounced rhinorrhea was only in $5 \%$ of patients.

The serum level of specific IgE antibodies to Alt a 1 significantly increased before the end of the 5th month of SIT (Fig.4b); at the same time the level of specific IgG antibodies has changed a little in this period of observation. At the end of the 5th month of SIT, it was noted an increase in the level of specific IgG antibodies to Alt a 1, which was associated with the reduced level of specific IgE antibodies (Fig.4c). At the end of treatment, the level of specific IgG antibodies was $29.7 \pm 8.2 \mathrm{kU} / 1(P<0.01)$, while the level of specific $\operatorname{IgE}$ antibodies decreased to $58 \pm 12.4 \mathrm{kU} / 1(P<0.01)$.

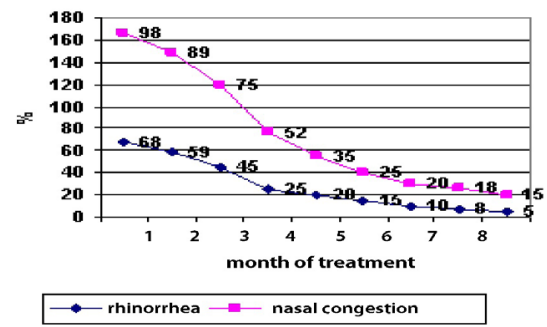

(a)

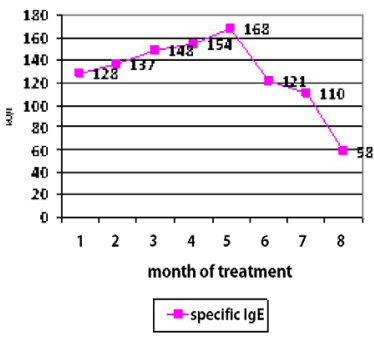

(b)

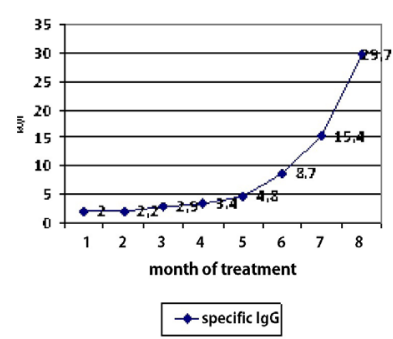

(c)
Fig.4. Dynamics of clinical manifestations (a), the level of specific $\operatorname{IgE}($ b) and $\operatorname{Ig} G(c)$ antibodies to Alt a 1 during SIT in PAR patients

Thus, a significant improvement in the clinical condition of patients during SIT was noted in both patient groups. In COPD patients, reduction of chronic cough and dyspnea on exertion was detected in $70 \%$ and $41 \%$, respectively. Among PAR patients, a disappearance of nasal congestion and rhinorrhea was observed in $83 \%$ and $63 \%$, respectively. The treatment induced the IgG response against Alt a 1. The concentration of specific IgG antibodies to Alt a 1 increased approximately 8-fold in COPD patients and 15-fold in PAR patients after 8 months of treatment. The mechanism of action of SIT is not definitively established, but it is known that the result of treatment-induced changes is the normalization of the immunological response. Immunologic changes that occur during allergen-specific immunotherapy are complex and not completely understood. However, successful immunotherapy has been associated with a shift from $\mathrm{T}$ helper cell type2 (Th2) immune responses, which are associated with the development of atopic conditions, to Th1 immune responses [16]. It is also associated with the production of $\mathrm{T}$ regulatory cells that produce the anti-inflammatory cytokine, IL-10, amongst others such as transforming growth factor (TGF)beta. IL-10 has been shown to reduce levels of allergenspecific IgE antibodies, increase levels of IgG (blocking) antibodies that play a role in secondary immune responses, and reduce the release of pro-inflammatory cytokines from mast cells, eosinophils and T cells [16-19]. Furthermore, there is an assumption that the allergen-specific IgG antibodies have the ability to reduce the early response to allergen, blocking the activation of FcE-dependent mast cells and releasing the carriers of perforin $[2,13]$.

Thus, our results indicate a positive effect of SIT on the clinical condition of COPD/PAR patients sensitized to Alternaria alternata. The clinical improvement was accompanied by a pronounced response of specific $\operatorname{IgG}$ antibodies to Alt a 1. However, research surrounding the mechanisms of immunotherapy is still ongoing and will help further elucidate how this form of therapy exerts its beneficial effects in patients sensitized to Alt a 1.

\section{Competing interests}

The authors declare that they have no competing interests.

\section{References}

1. Żukiewicz-Sobczak WA. The role of fungi in allergic diseases. Postepy Dermatol Alergol 2013; 30(1):42-45.

2. Asturias JA, Ibarrola I, Ferrer A, Andreu C, LopezPascual E, Quiralte J, et al. Diagnosis of Alternaria alternata sensitization with natural and recombinant Alt a 1allergens. J Allergy Clin Immunol 2005; 115:1210-7.

3. Andersson M, Downs S, Mitakakis T, Leuppi J, Marks G. Natural exposure to Alternaria spores induces allergic rhinitis symptoms in sensitized children. Pediatr Allergy Immunol 2003; 14(2):100-5.

4. Aden E, Weber B, Bossert J, Teppke M, Frank E, Wahl R et al. Standardization of Alternaria alternata: Extraction and quantification of Alt a 1 by using an $\mathrm{mAb}$-based 2-site binding assay. J Allergy Clin Immunol 1999; 104:128-35.

5. Mokhtari Amirmajdi M, Mokhtari Amirmajdi NA, Eftekharzadeh Mashhadi I, Jabari Azad F, Tavakol Afshari J, Shakeri MTIran. Alternaria in patients with allergic rhinitis. J Allergy Asthma Immunol 2011; 10(3):221-6.

6. Samaha HMS, Elsaid AR, NasrEldin E. Total serum IgE level in COPD patients. Egypt $\mathrm{J}$ of Chest Diseas Tubercul 2015; doi:10.1016/j.ejcdt.2015.02.005

7. Jamieson DB, Matsui EC, Belli A, McCormack MC, 
Peng E, Pierre-Louis S, et al. Effects of allergic phenotype on respiratory symptoms and exacerbations in patients with chronic obstructive pulmonary disease Am. J. Respir. Crit. Care Med., 188 (2013), pp. 187-192

8. Bourdin A, Serre I, Flamme H, Vic P, Neveu D, Aubas $\mathrm{P}$, et al.Can endobronchial biopsy analysis be recommended to discriminate between asthma and COPD in routine practice? Thorax, 59 (2004), pp. 488-493.

9. Jin J, Liu X, and Sun Y. The prevalence of increased serum IgE and Aspergillus sensitization in patients with COPD and their association with symptoms and lung function. Respiratory Research 2014, 15:130. doi:10.1186/s12931-014-0130-1

10. Han MK, Agusti A, Calverley PM, Celli BR, Criner G, Curtis JL, et al. Chronic obstructive pulmonary disease phenotypes: the future of COPD. Am J Respir Crit Care Med 2010, 182:598-604.

11. Jamieson DB, Matsui EC, Belli A, McCormack MC, Peng E, Pierre-Louis S, Curtin-Brosnan J, Breysse PN, Diette GB, Hansel NN: Effects of allergic phenotype on respiratory symptoms and exacerbations in patients with chronic obstructive pulmonary disease. Am J Respir Crit Care Med 2013, 188:187-192.

12. Celli BR, MacNee W, and committee members. Standards for the diagnosis and treatment of patients with COPD: a summary of the ATS/ERS position paper. Eur Respir J 2004;
23: 932-946.

13. Rohde G, Gevaert P, Holtappels G, Borg I, Wiethege A, Arinir $U$ et al. Increased IgE-antibodies to Staphylococcus aureusenterotoxins in patients with COPD. Respiratory Medicine 2004; 98:858-64.

14. Cortellini G, Spadolini I, Patella V, Fabri E, Santucci A, Severino M. Sublingual immunotherapy for Alternariainduced allergic rhinitis: a randomized placebo-controlled trial. Ann of Allergy, Asthma and Immunol 2010; 105(5):382-86. 15. Wachholz P.A, Durham S.R. Mehanisms of immunotherapy: IgG revizited. Cur Opin Allergy Clin Immun 2004; 4(4):313-18.

16. Moote W, Kim H. Allergen-specific immunotherapy. Allergy Asthma Clin Immunol. 2011; 7(Suppl 1): S5. doi: 10.1186/1710-1492-7-S1-S5.

17. Frew AJ. Allergen immunotherapy. J Allergy Clin Immunol 2010; 125 (2 Suppl 2):S306-13.

18. Canadian Society of Allergy and Clinical Immunology. Immunotherapy Manual. Fall. 2010.

19. Allergen immunotherapy: a practice parameter second update. Joint Task Force on Practice Parameters; American Academy of Allergy, Asthma and Immunology; American College of Allergy, Asthma and Immunology; Joint Council of Allergy, Asthma and Immunology. J Allergy Clin Immunol 2007; 120(3 Suppl):S25-85. 